\title{
Toeplitz-Based Iterative Image Reconstruction for MRI With Correction for Magnetic Field Inhomogeneity
}

\author{
Jeffrey A. Fessler, Senior Member, IEEE, Sangwoo Lee, Student Member, IEEE, \\ Valur T. Olafsson, Student Member, IEEE, Hugo R. Shi, Student Member, IEEE, and Douglas C. Noll, Member, IEEE
}

\begin{abstract}
In some types of magnetic resonance (MR) imaging, particularly functional brain scans, the conventional Fourier model for the measurements is inaccurate. Magnetic field inhomogeneities, which are caused by imperfect main fields and by magnetic susceptibility variations, induce distortions in images that are reconstructed by conventional Fourier methods. These artifacts hamper the use of functional MR imaging (fMRI) in brain regions near air/tissue interfaces. Recently, iterative methods that combine the conjugate gradient (CG) algorithm with nonuniform FFT (NUFFT) operations have been shown to provide considerably improved image quality relative to the conjugate-phase method. However, for non-Cartesian k-space trajectories, each CG-NUFFT iteration requires numerous k-space interpolations; these are operations that are computationally expensive and poorly suited to fast hardware implementations. This paper proposes a faster iterative approach to field-corrected MR image reconstruction based on the CG algorithm and certain Toeplitz matrices. This CG-Toeplitz approach requires k-space interpolations only for the initial iteration; thereafter, only fast Fourier transforms (FFTs) are required. Simulation results show that the proposed CG-Toeplitz approach produces equivalent image quality as the CG-NUFFT method with significantly reduced computation time.
\end{abstract}

Index Terms-fMRI imaging, magnetic susceptibility, non-Cartesian sampling, spiral trajectory.

\section{INTRODUCTION}

I $\mathrm{N}$ magnetic resonance (MR) imaging, the standard model for the measurements $\boldsymbol{y}=\left(y_{1}, \ldots, y_{M}\right)$ is

$$
E\left[y_{i}\right]=\int f(\vec{r}) \mathrm{e}^{-\imath 2 \pi \vec{\nu}_{i} \cdot \vec{r}} \mathrm{~d} \vec{r}, \quad i=1, \ldots, M
$$

where $f(\vec{r})$ denotes the unknown object magnetization, $\vec{r}$ denotes two-dimensional or three-dimensional (2-D or 3-D) spatial coordinates, $\vec{\nu}_{i}$ denotes the (possibly nonuniform) frequency-space sample locations associated with the specific MR

Manuscript received September 29, 2004; revised January 28, 2005. This work was supported in part by the National Institutes of Health unger Grant NIDA R01 DA15410. The associate editor coordinating the review of this paper and approving it for publication was Dr. Jan C. de Munck.

J. A. Fessler, S. Lee, V. T. Olafsson, and H. R. Shi are with the Department of Electrical Engineering and Computer Science, The University of Michigan, Ann Arbor, MI 48109-2122 USA (e-mail: fessler@umich.edu; sangwool@umich.edu; volafsso@umich.edu; hugoshi@umich.edu).

D. C. Noll is with the Biomedical Engineering Department, The University of Michigan, Ann Arbor, MI 48109-2099 USA (e-mail: dnoll@umich.edu).

Digital Object Identifier 10.1109/TSP.2005.853152 pulse sequence, and $E[\cdot]$ denotes expectation. MR measurements contain additive white complex Gaussian noise [1, Ch. $15]$ :

$$
y_{i}=E\left[y_{i}\right]+\varepsilon_{i}, \quad i=1, \ldots, M .
$$

The goal is to reconstruct $f(\vec{r})$ from $\boldsymbol{y}$.

The usual Fourier model (1) is reasonable for some types of MR scans, and many MR reconstruction methods are based on that model.

For MR scans with long readout times, there are off-resonance effects that are caused by magnetic field inhomogeneity (main field imperfections and magnetic susceptibility variations) and/or relaxation effects that depart from the simple Fourier model. Failure to compensate for such effects leads to geometric distortions in echo-planar imaging and blurring and artifacts when imaging with non-Cartesian trajectories. These degradations can be severe in brain scans based on the BOLD effect [2], hampering the use of fMRI in brain regions near air/tissue interfaces. Numerous solutions have been proposed based both on data acquisition strategies and reconstruction methods [3]-[22].

In the presence of such non-Fourier effects, a more realistic model for MR measurements is the following:

$$
E\left[y_{i}\right]=\int f(\vec{r}) \mathrm{e}^{-z(\vec{r}) t_{i}} \mathrm{e}^{-\imath 2 \pi \vec{\nu}_{i} \cdot \vec{r}} \mathrm{~d} \vec{r}
$$

where $t_{i}$ denotes the time of the $i$ th sample. The complex quantity $z(\vec{r})$ can include both relaxation and off-resonance effects as follows:

$$
z(\vec{r})=\alpha(\vec{r})+\imath \omega(\vec{r}) .
$$

The real function $\alpha(\vec{r})$ corresponds to the relaxation term (e.g., an $R_{2}^{*}$ map) at spatial position $\vec{r}$, and the real function $\omega(\vec{r})$ corresponds to off-resonance effects (e.g., susceptibility). Since both $\alpha(\vec{r})$ and $\omega(\vec{r})$ have inverse time units, we refer to $z(\vec{r})$ as the rate map hereafter. For simplicity here, we address the problem where the rate map $z(\vec{r})$ is known, i.e., where we are given relaxation maps $\alpha(\vec{r})$ and field maps $\omega(\vec{r})$, and the goal is to reconstruct the object $f$ from the measurements $\boldsymbol{y}$, e.g., [21]. For field-corrected MR reconstruction, usually one assumes that $\alpha(\vec{r})$ is zero. Further applications of the general approach described here include situations where either the field map $\omega(\vec{r})$ 
is unknown and must be estimated, e.g., [23]-[25], or the relaxation map $\alpha(\vec{r})$ is also to be estimated, e.g., [26], [27] or both, e.g., [28]-[35]. We focus on the case of a single receive coil, although the methods extend readily to parallel imaging with multiple coils, e.g., [36].

The standard approach to correcting these effects is the conjugate-phase image reconstruction method and its fast variants, e.g., [5], [37]. That family of methods is relatively fast since it is noniterative, but it only partially compensates for off-resonance effects. Recently, iterative methods that combine the conjugate gradient (CG) algorithm with nonuniform FFT (NUFFT) operations have been shown to provide considerably improved image quality relative to the conjugate-phase method [21]. However, for non-Cartesian k-space trajectories such as spirals, each CG-NUFFT iteration requires numerous k-space interpolations, which are also known as "gridding," e.g., [38]. These operations are computationally expensive and poorly suited to fast hardware implementations.

This paper proposes a faster iterative approach to field-corrected MR image reconstruction based on the CG algorithm and certain Toeplitz matrices. This CG-Toeplitz approach requires $\mathrm{k}$-space interpolations only for the initial iteration; thereafter, only fast Fourier transforms (FFTs) are required, making the method more suitable for fast hardware implementations. In the absence of field inhomogeneity, this method is closely related to certain algorithms for bandlimited signal interpolation, e.g., [39]. The Toeplitz/FFT structure has been investigated previously for MR image reconstruction in the context of sensitivity encoded imaging [40], [41]. The primary contribution here is the extension of such methods to the non-Fourier model (3). Simulation results with a realistic brain field map show that the proposed CG-Toeplitz approach significantly reduces computation time, yet produces image quality equivalent to the CG-NUFFT method.

The outline of this paper is as follows. Section II describes the basic CG approaches for iterative MR image reconstruction. Section III compares approximation methods for the nonFourier exponential $\mathrm{e}^{-z(\vec{r}) t_{i}}$ in (3). Section IV applies one of those approximations to derive the CG-Toeplitz method. Section $\mathrm{V}$ presents simulation results, showing the efficiency of the proposed approach.

\section{REgULARIZED LS RECONSTRUCTION}

\section{A. Object Discretization}

Equation (3) is a continuous-to-discrete model that is challenging to manipulate (see [42] and [43]). The problem is simplified by parameterizing the object $f(\vec{r})$ using a linear combination of $N$ basis functions:

$$
f(\vec{r})=\sum_{j=1}^{N} x_{j} p\left(\vec{r}-\vec{r}_{j}\right) .
$$

Therefore, the image reconstruction problem becomes that of estimating the parameter vector $\boldsymbol{x}=\left(x_{1}, \ldots, x_{N}\right)$ of expansion coefficients. For simplicity, we focus on rect functions (the voxel basis), as in [21], in which case, $N$ is the number of pixels, e.g., $64^{2}$, and $x_{j}$ is the $j$ th pixel value. We also assume that the rate map has (approximately) constant values over each voxel; therefore, we can write

$$
z(\vec{r})=\sum_{j=1}^{N} z_{j} p\left(\vec{r}-\vec{r}_{j}\right)
$$

where

$$
z_{j} \triangleq \alpha(\vec{r})+\imath \omega(\vec{r}), \quad j=1, \ldots, N .
$$

For cases with large within-voxel gradients of the rate map, one can use smaller voxels to reduce signal loss, albeit with increased computation [44], [45, p. 140].

Under these assumptions, the integral signal model (3) simplifies to the following discrete-to-discrete sum: ${ }^{1}$

$$
\bar{y}_{i}(\boldsymbol{x})=E\left[y_{i}\right]=P_{i} \sum_{j=1}^{N} x_{j} \mathrm{e}^{-z_{j} t_{i}} \mathrm{e}^{-\imath 2 \pi \vec{\nu}_{i} \cdot \vec{r}_{j}}
$$

using the following Fourier transform:

$$
P_{i} \triangleq P\left(\vec{\nu}_{i}\right)=\int p(\vec{r}) \mathrm{e}^{-i 2 \pi \vec{\nu}_{i} \cdot \vec{r}} \mathrm{~d} \vec{r}
$$

In matrix-vector form

$$
\begin{aligned}
\overline{\boldsymbol{y}}(\boldsymbol{x}) & =\boldsymbol{A} \boldsymbol{x}, \quad \boldsymbol{A}=\left\{a_{i j}\right\} \\
a_{i j} & =P_{i} \mathrm{e}^{-z_{j} t_{i}} \mathrm{e}^{-\imath 2 \pi \vec{\nu}_{i} \cdot \vec{r}_{j}} .
\end{aligned}
$$

Typically, the matrix $A$ is too large to be stored explicitly; therefore, we would like to use procedures like FFT operations to evaluate $\boldsymbol{A x}$, rather than explicit matrix-vector multiplication. Unfortunately, $\boldsymbol{A}$ is not a Fourier matrix in general. In any case, the MR reconstruction problem is to reconstruct $\boldsymbol{x}$ from $\boldsymbol{y}$ using (9).

\section{B. Regularized LS Minimization}

Since MR measurements have white complex Gaussian noise, we focus on methods that form an estimate $\hat{\boldsymbol{x}}$ of $\boldsymbol{x}$ by minimizing regularized least-squares cost functions of the form ${ }^{2}$

$$
\Psi(\boldsymbol{x})=\frac{1}{2}\|\boldsymbol{y}-\overline{\boldsymbol{y}}(\boldsymbol{x})\|^{2}+R(\boldsymbol{x})
$$

where $R(\boldsymbol{x})$ denotes any differentiable roughness penalty function, and $y$ denotes the measured data defined in (2). The goal is to find the image $\hat{x}$ that minimizes this cost function, typically by using gradient-based iterative algorithms. Most of the work in such algorithms is in computing the gradient of $\Psi$, and we focus on this computation hereafter.

One way to write the gradient of $\Psi$ is

$$
\nabla \Psi(\boldsymbol{x})=-\boldsymbol{A}^{\prime}(\boldsymbol{y}-\boldsymbol{A} \boldsymbol{x})+\nabla R(\boldsymbol{x})
$$

\footnotetext{
${ }^{1}$ In problems where $z_{j}$ is estimated by linearization, an extra " $t_{i}$ " term appears in the summation [35]. One can absorb this into $P_{i}$, and then, all remaining formulae are also applicable to such problems.

${ }^{2}$ An unweighted norm is used in the usual case where the measurements have equal variances, although the approach generalizes readily to weighted norms.
} 
where $\boldsymbol{A}^{\prime}$ denotes the adjoint (complex conjugate transpose) of $\boldsymbol{A}$. The computational bottleneck in (12) is calculating the matrix-vector products $\boldsymbol{A x}$ and $\boldsymbol{A}^{\prime} \boldsymbol{r}$, where $\boldsymbol{r}$ denotes the residual $\boldsymbol{y}-\boldsymbol{A x}$. We previously used the above gradient expression and combined NUFFTs [46] with temporal interpolation based on a "time-segmentation" approximation [5] so as to compute efficiently $\boldsymbol{A x}$ and $\boldsymbol{A}^{\prime} \boldsymbol{r}$ [21]. We refer to (12) as the "NUFFT approach."

An alternative, mathematically equivalent gradient expression is the following:

$$
\nabla \Psi(\boldsymbol{x})=\boldsymbol{T} \boldsymbol{x}-\boldsymbol{b}+\nabla R(\boldsymbol{x})
$$

where $\boldsymbol{T} \triangleq \boldsymbol{A}^{\prime} \boldsymbol{A}$, and $\boldsymbol{b} \triangleq \boldsymbol{A}^{\prime} \boldsymbol{y}$. Since $\boldsymbol{T}$ is Toeplitz when the rate map $z$ is zero, with some abuse of terminology, we refer to (13) as the "Toeplitz approach." The primary bottleneck in using (13) is multiplication of $\boldsymbol{T}$ by $\boldsymbol{x}$ for each iteration. If $\boldsymbol{T}$ were Toeplitz, then this could be done efficiently using well-known FFT methods [47], as has been proposed previously for iterative MR image reconstruction [40], [41]. Here, $\boldsymbol{T}$ is not Toeplitz due to the rate map $z$, so we will introduce approximations.

The next section first examines the approximations that have been used to evaluate (12). Section IV then returns to methods for computing efficiently the gradient expression (13).

\section{APPROXIMATIONS FOR EXPONENTIALS}

In the expression (10) for the elements of the matrix $\boldsymbol{A}$, the problematic part is the non-Fourier exponential terms $\mathrm{e}^{-z_{j} t_{i}}$. Direct implementation of $\boldsymbol{A} \boldsymbol{x}$ using (8) would require $O(M N)$ computations, which is undesirably slow. To reduce computation, one must make approximations, but these must be sufficiently accurate.

All of the known approximations are special cases of the following general form:

$$
\mathrm{e}^{-z_{j}} t_{i} \approx \sum_{l=1}^{L} b_{i l} c_{l j}, \quad \begin{aligned}
& j=1, \ldots, N \\
& i=1, \ldots, M
\end{aligned}
$$

for various choices for the $b_{i l}$ and $c_{l j}$ terms. Substituting such an approximation into the discrete signal model (8) and rearranging yields

$$
[\boldsymbol{A} \boldsymbol{x}]_{i} \approx P_{i} \sum_{l=1}^{L} b_{i l}\left[\sum_{j=1}^{N}\left(x_{j} c_{l j}\right) \mathrm{e}^{-\imath 2 \pi \vec{\nu}_{i} \cdot \vec{r}_{j}}\right] .
$$

In matrix form

$$
\boldsymbol{A} \approx \operatorname{diag}\left\{P_{i}\right\} \sum_{l=1}^{L} \operatorname{diag}\left\{b_{i l}\right\} \boldsymbol{G} \operatorname{diag}\left\{c_{l j}\right\}
$$

where $\boldsymbol{G}$ denotes the $M \times N$ NUFFT operator having elements $g_{i j}=\mathrm{e}^{-i 2 \pi \vec{\nu}_{i} \cdot \vec{r}_{j}}$, and $\operatorname{diag}\left\{P_{i}\right\}$ denotes a diagonal matrix with diagonal elements $\left\{P_{i}\right\}$. We can evaluate (15) efficiently using $L$ NUFFT calls [46] since the bracketed expression is an NUFFT of the signal $\left(x_{1} c_{l 1}, \ldots, x_{N} c_{l N}\right)$. In short, an approximation of the form (14) reduces computation since it contains no terms that depend on both $i$ and $j$.
Each NUFFT requires $O(K \log N)+O\left(J^{d} M\right)$, where $K$ is the over-sampled FFT size (typically $K=2^{d} N$ for $d$-dimensional imaging), and $J$ is the frequency domain interpolator width (typically $J=6$ ) [46]. Therefore, computing $A x$ via (15) reduces the total count from $O(M N)$ to $O(L(c N \log N+$ $\left.J^{d} M\right)$ ) for a small constant $c$.

The remainder of the section summarizes and compares possible choices for the $b_{i l}$ and $c_{l j}$ terms, including efficient methods for computing those terms.

\section{A. Time Segmentation (TS) Approximations}

In the context of MR reconstruction with field inhomogeneity correction, Noll et al. evaluated the exponentials $\mathrm{e}^{-z_{j} t_{i}}$ at a predetermined set of time points $\left\{\check{t}_{l}: l=1, \ldots, L\right\}$ and then used a linear interpolation method for times between those points [5], [37]. We can express this " time segmentation" approach as an approximation of the form (14), where

$$
b_{i l} \triangleq b_{l}\left(t_{i}\right) \mathrm{e}^{-\bar{z} t_{i}}, \quad c_{l j} \triangleq \mathrm{e}^{-\left(z_{j}-\bar{z}\right) \check{t}_{l}} .
$$

Each $b_{l}(t)$ denotes a temporal interpolator, and $\bar{z}$ denotes an (optional) baseline rate map value.

Originally, shift-invariant temporal interpolators were used [5]. These were generalized to min-max optimal temporal interpolators in [21], significantly reducing approximation error. (See Section III-F below.)

If one chooses $\bar{z}=0$, then the choice (16) reverts to the classical time segmentation method. Alternatively, if $z(\vec{r})$ is uniform with value $\bar{z}$, then (16) becomes exact if we choose $L=1$ and $b_{l}\left(t_{i}\right)=1$. A baseline $\bar{z}$ is useful for conventional interpolators but is not needed for the LS time-segmentation method described in (21) below.

\section{B. Frequency Segmentation}

Instead of choosing time samples, an alternative approach is to choose a set of "frequency" samples $\breve{z}_{l}$, for $l=1, \ldots, L$, and interpolate between these values to evaluate the exponential [37], [48], [49]. We can express this "frequency segmentation" approach as an approximation of the form (14) with

$$
b_{i l} \triangleq \mathrm{e}^{-\check{z}_{l}\left(t_{i}-\bar{t}\right)}, \quad c_{l j} \triangleq c_{l}\left(z_{j}\right) \mathrm{e}^{-z_{j} \bar{t}}
$$

where $\bar{t}$ is a nominal time reference (e.g., an echo time, or simply $\bar{t}=0$ ), and where each $c_{l}(\cdot)$ denotes a frequency-domain interpolator.

In the original version [37], the $c_{l j}$ 's were chosen to be either nearest-neighbor, linear, or Hanning interpolators. (See also [20].) Later, Man et al. described a least-squares approach [cf. (19) below] to choosing the interpolators $c_{l}(\cdot)$ [49].

In the frequency segmentation approach, a practical issue is choosing the frequency samples $\left\{\check{z}_{l}\right\}$. The traditional choice is equally spaced frequencies that span the bandwidth of the field map. However, that choice is suboptimal for nonuniform field map distributions. Instead, it is preferable to concentrate more frequency components where they are most needed based on the rate map histogram. We achieve this by using the asymptotic theory of quantization, which specifies the optimal density of centroids for high-rate quantization [50]. 


\section{Generalized Approximations}

Both "time segmentation" and "frequency segmentation" lead to approximations of the form (14), and both enable the efficient implementation (15). Thus, from the point of view of rapid computation, time segmentation and frequency segmentation are equally viable methods. In fact, for a given $L$, any choices for the $b_{i l}$ and $c_{l j}$ terms lead to the same compute time for evaluating $\boldsymbol{A x}$.

Since computing times are determined only by $L$ (and $N$ and $M)$, rather than by the form of $b_{i l}$ and $c_{l j}$, it is natural to consider choosing the $b_{i l}$ and $c_{l j}$ terms to minimize the error in the approximation (14). Let $\boldsymbol{B}=\left\{b_{i l}\right\} \in \mathbb{C}^{M \times L}$ and $\boldsymbol{C}=\left\{c_{l j}\right\} \in$ $\mathbb{C}^{L \times N}$. We would like to examine choices for $B$ and $C$ that are "optimal" in some sense, without necessarily being constrained to the exponential forms used in (16) and (17).

The possibility of using nonexponential bases was explored in [49] using SVD analysis, with the conclusion that frequency segmentation is nearly optimal. However, that investigation used equally weighted, equally spaced frequency samples, which corresponds implicitly to rate maps having uniform distributions (a rectangular histogram). In practice, the rate maps for real brain scans can be quite nonuniform.

The least-squares optimal choices for $B$ and $C$ minimize the Frobenius norm

$$
\begin{aligned}
& \underset{\boldsymbol{B}, \boldsymbol{C}}{\arg \min }\|\boldsymbol{E}-\boldsymbol{B} \boldsymbol{C}\|_{\text {Frob }}^{2} \\
& \quad=\underset{\boldsymbol{B}, \boldsymbol{C}}{\arg \min } \sum_{i=1}^{M} \sum_{j=1}^{N}\left|\mathrm{e}^{-z_{j} t_{i}}-\sum_{l=1}^{L} b_{i l} c_{l j}\right|^{2}
\end{aligned}
$$

or a weighted generalization thereof, where $\boldsymbol{E}$ is the $M \times N$ matrix with elements $e_{i j}=\mathrm{e}^{-z_{j} t_{i}}$. This minimization is a "principal components" problem that is solved by the SVD of $\boldsymbol{E}$. This solution can be of theoretical interest as a performance benchmark but appears to require too much memory and computation for routine use.

Rather than optimizing both $\boldsymbol{B}$ and $\boldsymbol{C}$ jointly, one can first choose $\boldsymbol{B}$ heuristically and then find the matrix $\boldsymbol{C}$ that optimizes (18), or one can first choose $\boldsymbol{C}$ and then optimize $\boldsymbol{B}$. These two alternatives are explored next.

\section{Histogram Principal Components}

For a given matrix $\boldsymbol{B}$, the LS-optimal choice of $\boldsymbol{C}$ is

$$
\boldsymbol{C}=\left[\boldsymbol{B}^{\prime} \boldsymbol{B}\right]^{-1} \boldsymbol{B}^{\prime} \boldsymbol{E}
$$

We now focus on choosing $B$ efficiently. To simplify (18), we histogram the rate map values $\left\{z_{j}\right\}$ into $K \ll N$ bins with centers $\tilde{z}_{k}, k=1, \ldots, K$, possibly spaced unequally, and let $h_{k}$ denote the number of $z_{j}$ values in the $k$ th bin. Then, a natural approximation to (18) is the following WLS criterion:

$$
\underset{\boldsymbol{B}}{\arg \min } \sum_{k=1}^{K} h_{k}\left\|\boldsymbol{e}_{k}-\boldsymbol{B}\left[\boldsymbol{B}^{\prime} \boldsymbol{B}\right]^{-1} \boldsymbol{B}^{\prime} \boldsymbol{e}_{k}\right\|^{2}
$$

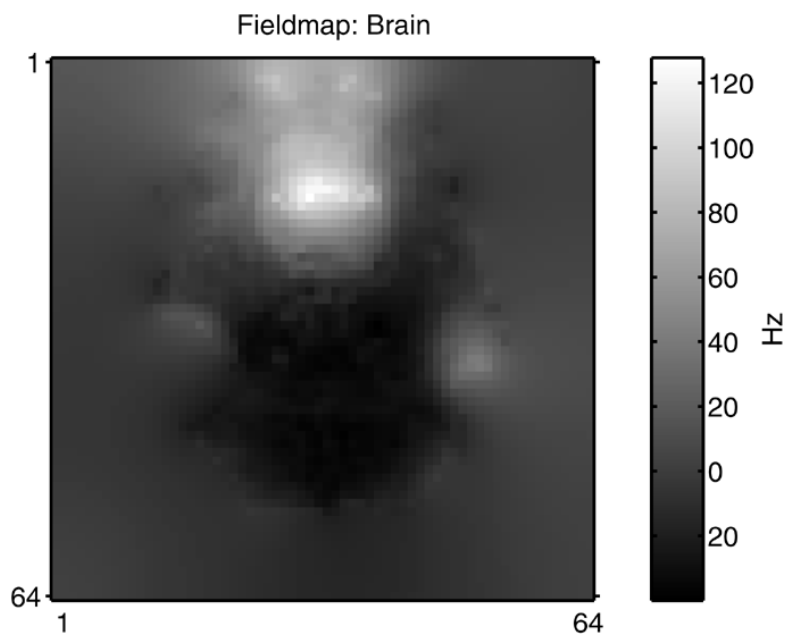

Fig. 1. Brain field map $\omega(\vec{r}) / 2 \pi$.

where we define $e_{k}=\left(\mathrm{e}^{-\tilde{z}_{k} t_{1}}, \ldots, \mathrm{e}^{-\tilde{z}_{k} t_{M}}\right)$. The solution to this minimization problem is given by the first $L$ left singular vectors of the $M \times K$ matrix $\left[\sqrt{h_{1}} \tilde{\boldsymbol{e}}_{1} \ldots \sqrt{h_{K}} \tilde{\boldsymbol{e}}_{K}\right]$. Since $K \ll$ $N$, this singular value dcompositoin (SVD) is much more practical than (18).

\section{E. LS Frequency-Segmentation Approach}

As described in [49], one can choose $\boldsymbol{B}$ using the frequency-segmentation choice (17) and then find the corresponding LS-optimal choice of $\boldsymbol{C}$ using (19).

\section{F. LS Time-Segmentation Approach}

To avoid SVDs altogether, a simpler approach is to choose the matrix $C$ that corresponds to the time segmentation approximation (16) and then optimize $\boldsymbol{B}$ by least squares [21]. (When $B$ is thus optimized, the $\bar{z}$ term in (16) is unnecessary.) Again, to reduce computation, we histogram the rate map values as described above [21]. Letting $\boldsymbol{b}\left(t_{i}\right)=\left(b_{1}\left(t_{i}\right), \ldots, b_{L}\left(t_{i}\right)\right)$ denote the $i$ th row of $\boldsymbol{B}$, we find $\boldsymbol{B}$ by the following WLS criterion:

$$
\boldsymbol{b}(t)=\underset{\boldsymbol{b} \in \mathbb{C}^{L}}{\arg \min } \sum_{k=1}^{K} h_{k}\left|\mathrm{e}^{-\tilde{z}_{k} t}-\sum_{l=1}^{L} b_{l} \mathrm{e}^{-\tilde{z}_{k} \check{t}_{l}}\right|^{2}
$$

where $h_{k}$ was defined before (20). For $K \ll N$ histogram bins, the computation of $\boldsymbol{B}$ is $O\left(L K(M+L)+L^{3} M\right)$.

\section{G. Comparisons}

We evaluated the above approximations for a wide variety of simulated and real fieldmaps. We summarize here one representative comparison, using the brain fieldmap shown in Fig. 1. This map (a brain slice near the ear canals) was acquired using standard delayed-echo field mapping methods on a GE 3T MR scanner [51]. Fig. 2 shows the histogram of this field map.

For evaluation, we used $t_{i}$ 's with $5 \mu$ s sampling for $M=$ 3770 , corresponding to a $18.855-\mathrm{ms}$ readout time. This time is typical for one-shot spiral trajectories on our 3T GE scanner for $64 \times 64$ brain scans with a $22-\mathrm{cm}$ field of view (FOV).

We compared three approximations: i) the SVD approach of Section III-D using the histogram approximation (20) with 


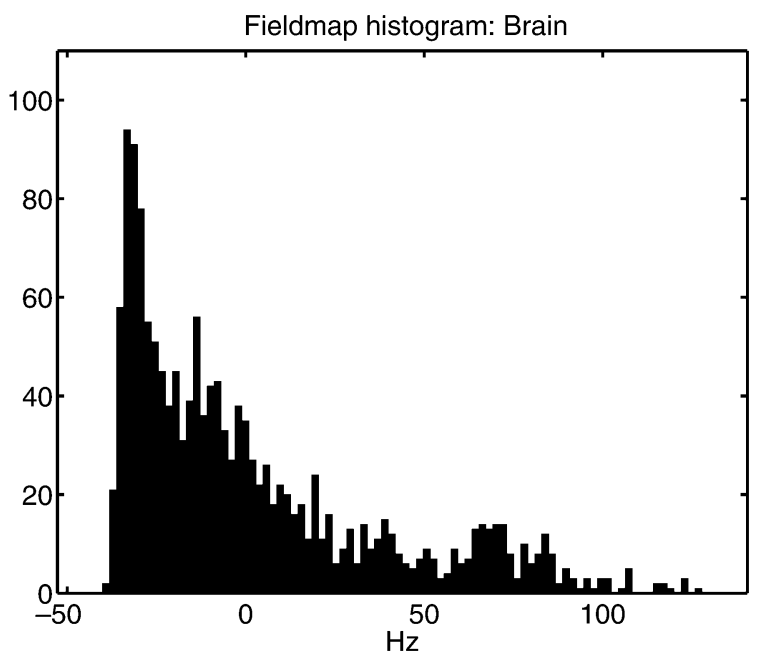

Fig. 2. Histogram of the fieldmap shown in Fig. 1.

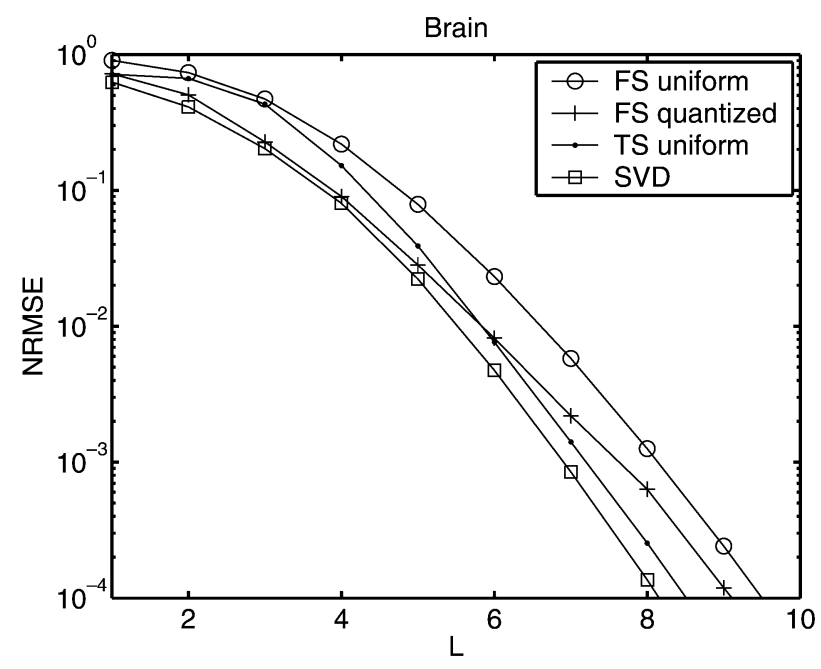

Fig. 3. Normalized RMS error for approximations to the exponentials $\mathrm{e}^{-z_{j} t_{i}}$ for the field map shown in Fig. 1.

$K=40$ bins; ii) the time-segmentation (TS) approach of Section III-F with the WLS criterion (21); iii) the frequency-segmentation (FS) method of Section III-E using the LS-optimal interpolators (19). For FS, we found that uniformly spaced $\check{z}_{l}$ values worked well only for a simple fieldmap that varied linearly over space, which has a uniform field histogram (results not shown). As an alternative, we applied the Lloyd-Max algorithm from scalar quantizer design to choose the frequency samples from the fieldmap histograms. This reduced error in all cases.

Fig. 3 shows the normalized root mean-squared error (NRMSE), which is defined by $1 / N\left|\|\boldsymbol{E}-\boldsymbol{B C} \mid\|_{\text {Frob }}\right.$ [see (18)], as a function of $L$ for the fieldmap shown in Fig. 1, for all four approximations. Naturally, as the number of approximation terms $L$ increases, the error decreases. In all cases, for any given $L$, the SVD approach has the minimum error. However, the TS approximation has only slightly larger error. In fact, to achieve a NRMSE less than 1\%, both the SVD and the TS methods require $L=6$ for this fieldmap.
From these representative results and others not shown, we conclude that TS approximations, when optimized per Section III-F, provide the most attractive tradeoff between accuracy and ease of computation. This conclusion is fortuitous since the Toeplitz approach described in Section IV is most efficient when implemented with TS approximations.

\section{TOEPLITZ APPROACH}

Now, we turn to computing the "Toeplitz approach" (13) efficiently. Under the model (9), the matrix $\boldsymbol{T}$ in (13) has the following elements:

$$
\begin{aligned}
T_{k j} & =\left[\boldsymbol{A}^{\prime} A\right]_{k j}=\sum_{i=1}^{M} a_{i k}^{*} a_{i j} \\
& =\sum_{i=1}^{M}\left|P_{i}\right|^{2} \mathrm{e}^{-\left(z_{k}^{*}+z_{j}\right) t_{i}} \mathrm{e}^{-\imath 2 \pi \vec{\nu}_{i} \cdot\left(\vec{r}_{j}-\vec{r}_{k}\right)} .
\end{aligned}
$$

In the usual case where the voxel centers $\vec{r}_{j}$ are spaced equally, this matrix would be Toeplitz ${ }^{3}$ in the absence of relaxation effects and off-resonance effects, i.e., when $z(\vec{r})=0$.

In the presence of such effects, $T$ is not Toeplitz due to the problematic term $\mathrm{e}^{-\left(z_{k}^{*}+z_{j}\right) t_{i}}$. Therefore, we must introduce approximations to develop fast methods for computing the matrix-vector product $\boldsymbol{T} \boldsymbol{x}$ required in the gradient calculation (13). Two possible approaches are described next.

\section{A. $O\left(L^{2}\right)$ Approach}

One approach is to separate the problematic exponential first and then make approximations as follows:

$$
\mathrm{e}^{-\left(z_{k}^{*}+z_{j}\right) t_{i}}=\mathrm{e}^{-z_{k}^{*} t_{i}} \mathrm{e}^{-z_{j} t_{i}} \approx\left(\sum_{l^{\prime}=1}^{L} b_{i l^{\prime}} c_{l^{\prime} k}\right)^{*} \sum_{l=1}^{L} b_{i l} c_{l j}
$$

i.e., to invoke approximations of the form (14) twice. Substituting into (22) and rearranging leads to the following:

$$
\boldsymbol{T} \approx \sum_{l^{\prime}=1}^{L} \sum_{l=1}^{L} \boldsymbol{D}_{l^{\prime}}^{\prime} \boldsymbol{T}_{l^{\prime}, l} \boldsymbol{D}_{l}
$$

where $\boldsymbol{D}_{l}=\operatorname{diag}\left\{c_{l j}\right\}$, and

$$
\left[\boldsymbol{T}_{l^{\prime}, l}\right]_{k j}=\sum_{i=1}^{n_{d}}\left|P_{i}\right|^{2} b_{i l^{\prime}}^{*} b_{i l} \mathrm{e}^{-\imath 2 \pi \vec{\nu}_{i} \cdot\left(\vec{r}_{j}-\vec{r}_{k}\right)} .
$$

Each matrix $\boldsymbol{T}_{l^{\prime}, l}$ is Toeplitz, and therefore, we can multiply this approximation to $T$ by a vector $x$ using $L^{2}$ pairs of FFTs [47]. An advantage of this approach is that one can use the $B$ and $C$ matrices corresponding to any exponential approximation (14). However, a significant disadvantage is that it requires $O\left(L^{2}\right)$ computation.

\section{B. $O(L)$ Approach}

To reduce computation, we would like to use an approximation for the problematic exponential term that will allow us to

${ }^{3}$ For simplicity, we say "Toeplitz" rather than "block Toeplitz with Toeplitz blocks" [47]. 
"separate" the $z_{k}^{*}+z_{j}$ term in (22) after making the approximation. Of the various approximation methods described in Section III, only the time segmentation approach appears to have the desired property. (Fortunately, the time segmentation approach is also sufficiently accurate, as shown in Section III-G.) Substituting the approximation (16) (with $\bar{z}=0$ ) into (22) yields the following approximation to the elements of $\boldsymbol{T}$ :

$$
\begin{aligned}
T_{k j} & \approx \sum_{i=1}^{M}\left|P_{i}\right|^{2}\left[\sum_{l=1}^{L} b_{i l} \mathrm{e}^{-\left(z_{k}^{*}+z_{j}\right) \check{t}_{l}}\right] \mathrm{e}^{-\imath 2 \pi \vec{\nu}_{i} \cdot\left(\vec{r}_{j}-\vec{r}_{k}\right)} \\
& =\sum_{l=1}^{L} \mathrm{e}^{-z_{k}^{*} \check{t}_{l}}\left[\boldsymbol{T}_{l}\right]_{k j} \mathrm{e}^{-z_{j} \check{t}_{l}}
\end{aligned}
$$

where the element of each matrix $\boldsymbol{T}_{l}$ is defined by

$$
\left[\boldsymbol{T}_{l}\right]_{k j}=\sum_{i=1}^{M}\left|P_{i}\right|^{2} b_{i l} \mathrm{e}^{-\imath 2 \pi \vec{\nu}_{i} \cdot\left(\vec{r}_{j}-\vec{r}_{k}\right)}
$$

In matrix form

$$
T \approx \sum_{l=1}^{L} D_{l}^{\prime} T_{l} D_{l}
$$

where $\boldsymbol{D}_{\boldsymbol{l}}=\operatorname{diag}\left\{\mathrm{e}^{-z_{j} \check{\check{t}}_{l}}\right\}$. Each matrix $\boldsymbol{T}_{l}$ is Toeplitz, and therefore, one can multiply $\boldsymbol{T}_{l}$ by a vector efficiently using a pair of FFTs [47]. These FFTs use the first row of $\boldsymbol{T}_{l}$, which we precompute prior to iterating by a pair of NUFFT calls. Each $\boldsymbol{D}_{l}$ matrix is diagonal; therefore, multiplying with it is trivial. Thus, to compute $\boldsymbol{T} \boldsymbol{x}$ (approximately) requires $L$ pairs of FFTs, for an operation count of $O(L N \log N)$. In contrast, the NUFFT approach that uses the gradient expression in (12) with an approximation like (15) requires $L$ pairs of NUFFTs, which is more computation due to interpolations [46].

A subtle but key issue in using (24) is choosing the interpolators $b_{i l}$. If the rate map $z_{j}$ contains frequency offsets in the range $\nu_{\min }$ to $\nu_{\max }$, then the term $\mathrm{e}^{-\left(z_{k}^{*}+z_{j}\right) t}$ will contain frequency offsets in the range $-\left(\nu_{\max }-\nu_{\min }\right)$ to $\nu_{\max }-\nu_{\min }$. In other words, its "bandwidth" is twice as wide as the bandwidth of $\mathrm{e}^{-z_{j} t}$. Therefore, we have found that it can be necessary to use larger values of $L$ for the Toeplitz approximation (24) than for the NUFFT approximation (15). Nevertheless, by avoiding discrete Fourier transform (DFT) interpolations, the Toeplitz approach is still faster than the NUFFT approach.

For (25) to be accurate, we would like to choose $\boldsymbol{B}$ to provide an LS approximation to terms of the form $\mathrm{e}^{-\left(z_{k}{ }^{*}+z_{j}\right) t_{i}}$. For a fieldmap with a given histogram $\left\{h_{k}\right\}$, the histogram of $z_{k}^{*}+z_{j}$ is given by the auto-correlation function of $h_{k}$. Therefore, to design $B$ for the Toeplitz approach, we first find the fieldmap histogram, then compute the auto-correlation function of that histogram, and then apply the WLS criterion (21) using that auto-correlated histogram. We found that this approach provided much improved accuracy relative to using (21) with the original histogram. Furthermore, because "auto-correlated" histograms are symmetric about zero, the resulting $\boldsymbol{B}$ matrix is real valued, saving computation in precomputing the Toeplitz kernels in (25).

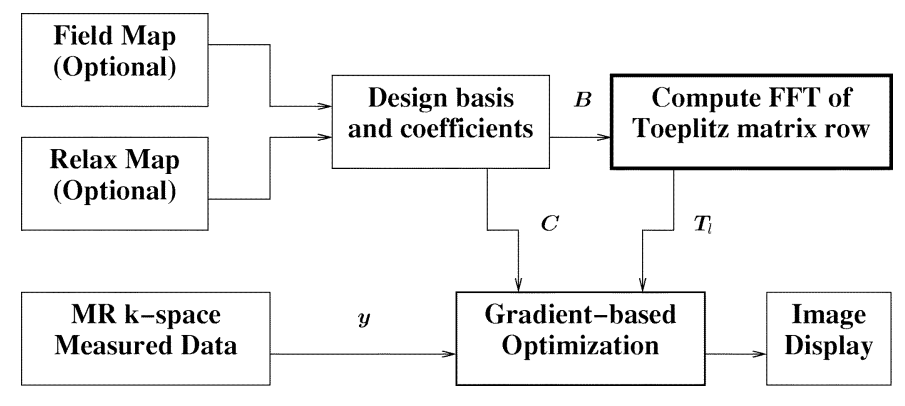

Fig. 4. Block diagram of MR image reconstruction data flow.

We summarize all of the required steps as follows. Fig. 4 illustrates the data flow. 4

\section{CG-Toeplitz Algorithm}

- Determine the relaxation map and/or the field map to form the rate map $z(\vec{r})$ in (4).

- Compute the histogram of that rate map and then the autocorrelation function of that histogram.

- Using that auto-correlated histogram, use (21) and (16) to compute the interpolators $\boldsymbol{B}$ and the coefficients $\boldsymbol{C}$ using the LS time-segmentation method of Section III-F.

- Precompute $\boldsymbol{b}=\boldsymbol{A}^{\prime} \boldsymbol{y}$ using the combination of temporal interpolation and NUFFT methods described in [21] and [46]. Since this need only be done once, rather than each iteration, it can be done with a high-accuracy approximation.

- Precompute the first row of $\boldsymbol{T}_{l}$ for $l=1, \ldots, L$ using (25), in preparation for using a $2 \times$ oversampled FFT to perform the operation of matrix-vector multiplication by $\boldsymbol{T}_{l}$ [47]. This requires $L$ pairs of NUFFT calls.

- Using (26) to compute $\boldsymbol{T} \boldsymbol{x}$ approximately for the gradient expression (13), apply a gradient-based optimization method such as the CG algorithm (e.g., [21]) to find $\hat{\boldsymbol{x}}$ iteratively.

\section{Simulation}

We compared four methods for field-corrected MR image reconstruction: i) the conjugate-phase reconstruction method [5] using Voronoi-based density compensation factors [52] and the LS-optimal time-segmentation approximation described in Section III-F, ii) the CG-NUFFT method based on the gradient expression (12), using the time-segmentation approximation described in Section III-F [21], iii) the CG-Toeplitz method based on the gradient expression (13) using the $O(L)$ approximation described in Section IV, and iv) for completeness, the conjugatephase method without field correction. For the CG methods, we used quadratic regularization with a small regularization parameter, chosen such that the FWHM of the PSF was about 1.36 pixels. For simplicity, we initialized the CG algorithms with $\boldsymbol{x}=\mathbf{0}$.

To evaluate the methods quantitatively, we performed simulations using the brain fieldmap shown in Fig. 1, and the synthetic image $\boldsymbol{x}$ shown in Fig. 5. We evaluated the reconstruction methods using a spiral trajectory containing 3770 points with a sampling time of $5 \mu$ s so that the data acquisition time was

\footnotetext{
${ }^{4}$ Software available on web site http://www.eecs.umich.edu/ fessler.
} 


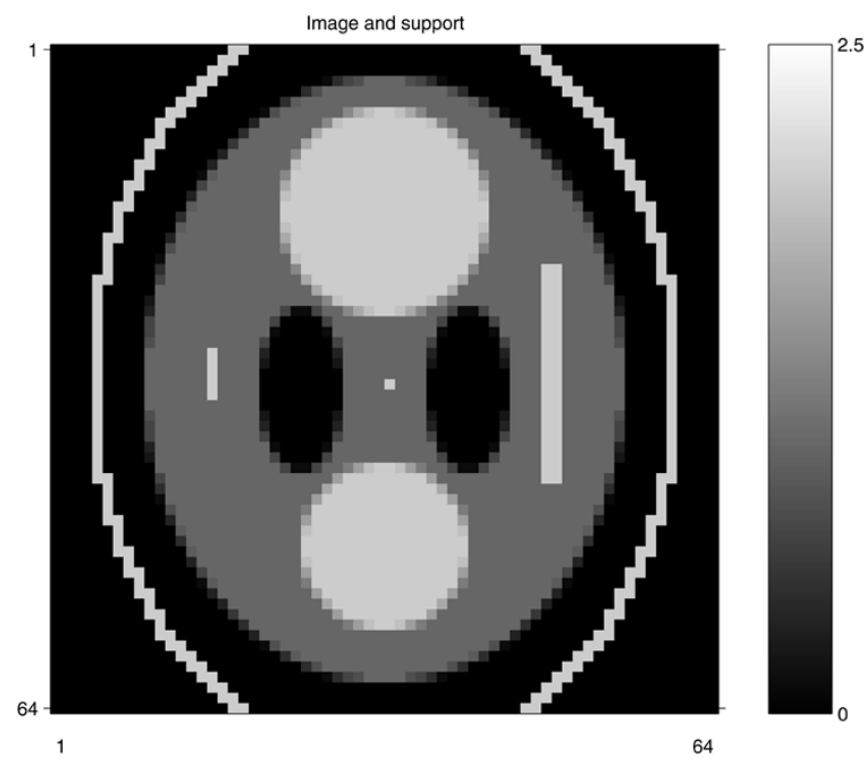

Fig. 5. True image $\boldsymbol{x}$ used in simulations. Only pixels within the outer elliptical region were reconstructed.

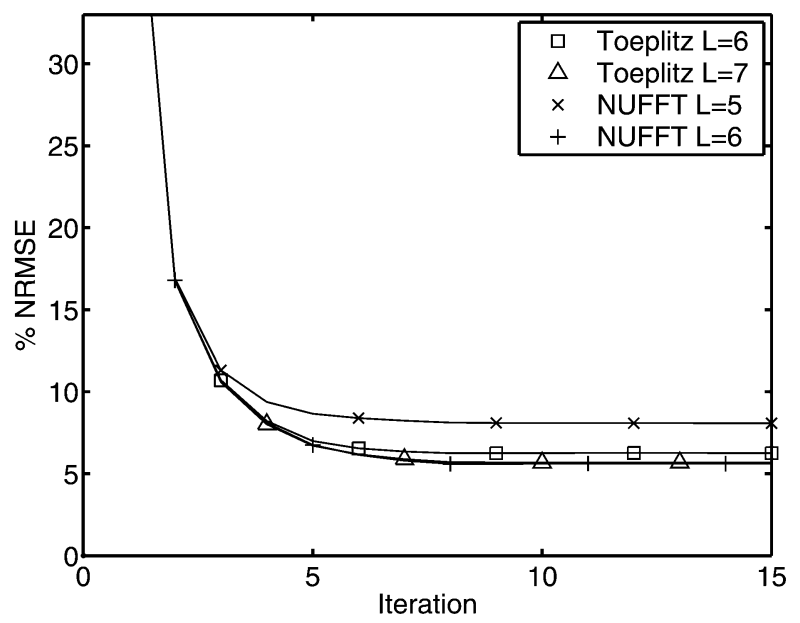

Fig. 6. NRMSE of $\hat{x}$ versus iteration for the two CG construction methods for the spiral trajectory.

$18.855 \mathrm{~ms}$. This spiral trajectory is used routinely on our GE 3T MR system. To generate the (noiseless) simulated data $\overline{\boldsymbol{y}}$, we used the exact system matrix (10).

For all methods, we estimated only the 2936 pixels within the elliptical region of interest shown in Fig. 5. For reconstruction, we used NUFFTs with $2 \times$ oversampling and $J=6$, which we have found previously to be sufficiently accurate.

Fig. 6 shows the NRMS error as a function of iteration, which is defined as $\left\|\hat{\boldsymbol{x}}-\boldsymbol{x}^{\text {true }}\right\| /\left\|\boldsymbol{x}^{\text {true }}\right\| \cdot 100 \%$ for the values of $L$ listed. Larger values of $L$ did not reduce the error further. Since there was no noise in the simulated $\mathrm{k}$-space data, the lower limit on NRMS error is a function of the (modest) regularization used and the inherent NUFFT approximations. For these values of $L$ (or larger), the CG algorithm essentially converged by 15 iterations.

Fig. 7 shows the NRMS error as a function of $L$. To achieve the same accuracy, the CG-Toeplitz approach requires $L$ to be slightly larger than for CG-NUFFT. The RMS error of the CP

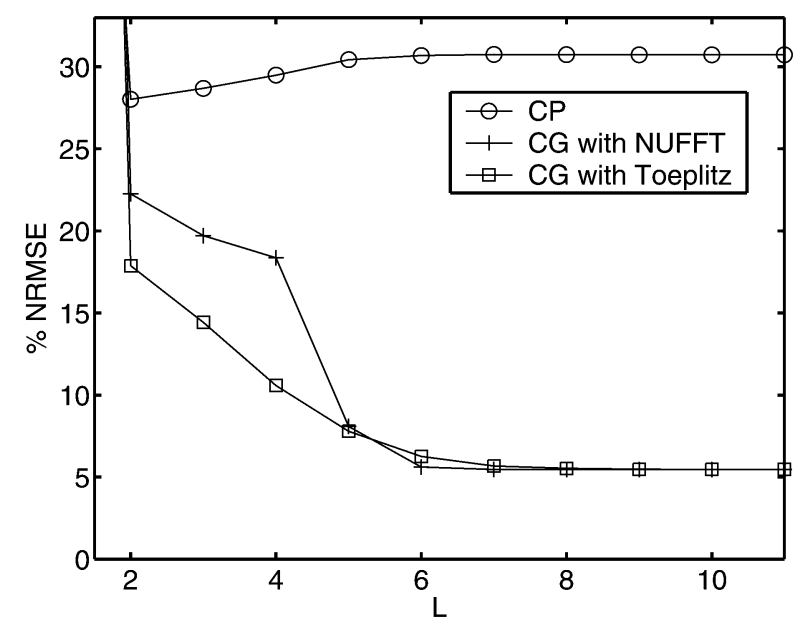

Fig. 7. NRMSE of $\hat{x}$ versus approximation order $L$ for the three field-corrected reconstruction methods for the spiral trajectory.

method changes relatively little for $L>1$, apparently because that error is dominated by imperfect density compensation for the spiral trajectory. We separately examined a Cartesian trajectory (results not shown), where density compensation is moot, and in that case, the NRMS error decreased monotonically in $L$ until reaching a minimum value of $14 \%$ at $L=6$.

Fig. 8 shows the reconstructed images. Based on the results in Fig. 7, we used $L=6$ for the conjugate phase and CG-NUFFT approaches and $L=8$ for the CG-Toeplitz approach.

Table I compares the CPU time of the various reconstruction methods (using MATLAB's cputime on a Dell $650 \mathrm{n}$ with 3.06 $\mathrm{GHz}$ Xeon CPU). For the CG methods, the times are for 15 iterations, which is adequate based on Fig. 6. The total times shown in the table include the time required to "precompute" $\boldsymbol{B}, \boldsymbol{C}$, etc. The Toeplitz approach shows significant acceleration. In MATLAB, for the same $L$, the Toeplitz approach runs several times faster per iteration than the NUFFT approach because it avoids the NUFFT interpolations. The Toeplitz approach requires a slightly larger value for $L$ and requires precomputing the kernels of the $\boldsymbol{T}_{l}$ terms, but despite this "overhead," the overall compute time is still reduced significantly.

To investigate whether the approximations would increase sensitivity to noise, we added several different levels of pseudo-random white complex Gaussian noise to $\overline{\boldsymbol{y}}$ and repeated the reconstructions. Table I shows that the noise properties of the CG-NUFFT and CG-Toeplitz approach are indistinguishable because the chosen $L$ values ensure that approximation error is negligible relative to estimation error.

\section{SUMMARY}

This paper has described a new CG-Toeplitz method for fieldcorrected MR image reconstruction using the approximations (26). Simulation results show that this proposed method is as accurate as the previously proposed CG-NUFFT method [21] but is considerably faster. The CG-Toeplitz approach is also better suited to fast hardware implementation since only FFTs are required during the iterations, eliminating the frequency domain interpolations required by the CG-NUFFT approach. We believe that the CG-Toeplitz approach is the method of choice 


\section{Uncorrected Conj. Phase, L=6}

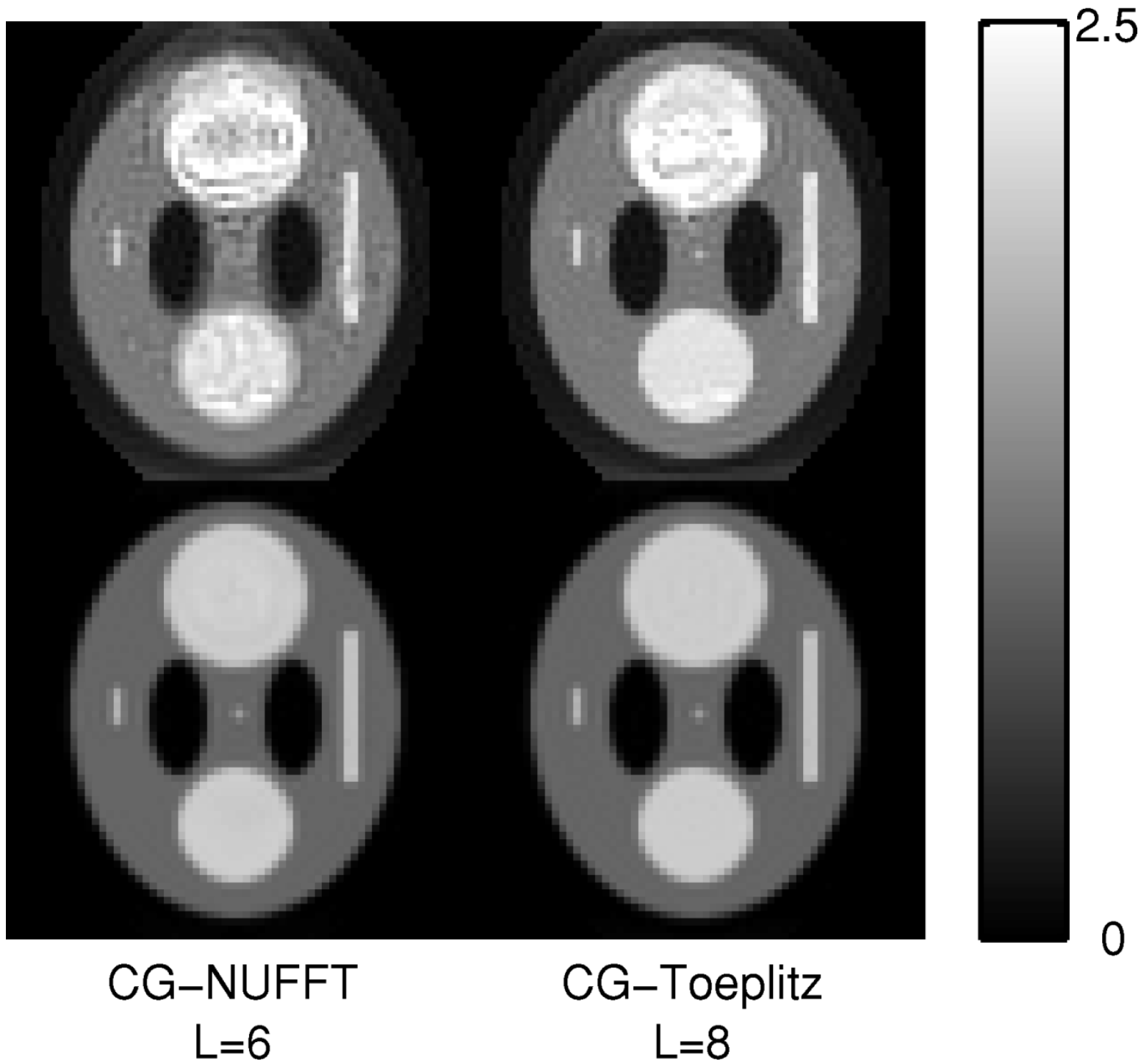

Fig. 8. Reconstructed images for the spiral trajectory.

TABLE I

CPU Times (IN SECONDS), InCluding PRECOMPUTATION TIMES AND NRMS ERROR (\%) FOR Three Field-CORRECTED MR IMAGE ReCONSTRUCTION Methods. The Proposed CG-TOEPLITZ APPROACH Is FASTER THAN CG-NUFFT Yet EQUALly ACCURATE

\begin{tabular}{|c|c|c|c|c|c|c|c|c|c|c|c|c|}
\hline & & \multicolumn{4}{|c|}{ Precomputation } & & & \multicolumn{5}{|c|}{ NRMS \% vs SNR } \\
\hline Method & $L$ & $B, C$ & $A^{\prime} D y$ & $b=A^{\prime} y$ & $\boldsymbol{T}_{l}$ & 15 iter & Total Time & $\infty$ & $50 \mathrm{~dB}$ & $40 \mathrm{~dB}$ & $30 \mathrm{~dB}$ & $20 \mathrm{~dB}$ \\
\hline Conj. Phase & 6 & 0.4 & 0.2 & & & & 0.6 & 30.7 & 37.3 & 46.5 & 65.3 & 99.9 \\
\hline CG-NUFFT & 6 & 0.4 & & & & 5.0 & 5.4 & 5.6 & 16.7 & 26.5 & 43.0 & 70.4 \\
\hline$\overline{\text { CG-Toeplitz }}$ & 8 & 0.4 & & 0.2 & 0.6 & 1.3 & 2.5 & 5.5 & 16.7 & 26.4 & 42.9 & $\overline{70.4}$ \\
\hline
\end{tabular}

for iterative field-corrected MR image reconstruction. The improved image quality in regions with severe field inhomogeneity may enable detection of brain activation, even in regions near air/tissue interfaces.

An alternative $\mathrm{CG}$ approach has recently been proposed by Barnet et al. [53]. That approach involves expressions of the form $\boldsymbol{A} \boldsymbol{A}^{\prime}$, which is never Toeplitz, even when the rate map is zero; therefore, it cannot benefit from the accelerations proposed here. Furthermore, it is limited to the special case of quadratic regularization with an invertible Hessian, whereas the gradient- based approach that uses (12) or (13) can accommodate even nonquadratic regularization methods, e.g., [54].

There are several opportunities to extend this work.

- When $z(\vec{r})=0$, the matrix $\boldsymbol{T}$ in (22) is Toeplitz, and good circulant preconditioners are available [47]. When $z(\vec{r}) \neq 0$, then $\boldsymbol{T}$ is approximately the "weighted sum" of Toeplitz matrices in (26). An open question for future work is how to precondition this sum effectively; preconditioners have been developed for other shift-variant problems [47], [55]. 
- The model (6) assumes that the rate map is constant over each voxel. To compensate for within-voxel field gradients, one can use smaller voxels [44]. This increases computation; therefore, an interesting challenge is to try to account for field gradients with less computation.

- For echo-planar imaging (EPI), the primary blur is in the readout direction. This affects the properties of the $\boldsymbol{T}_{l}$ matrices, and it may be possible to further reduce computation.

- For both the NUFFT and Toeplitz methods investigated here, we used FFTs with $2 \times$ oversampling in each dimension. In the absence of field inhomogeneity, NUFFT-type methods may tolerate smaller oversampling factors [41]. Whether the Toeplitz approach could also tolerate reduced oversampling requires further investigation, particularly in the presence of field inhomogeneity.

- For the methods described here, we separated the problems of designing the "temporal" interpolators $\boldsymbol{B}$ and $\boldsymbol{C}$ and of designing the interpolators that are used in the frequency domain for the NUFFT operation. Whether one could design both interpolators simultaneously to improve accuracy (or reduce computation) is an interesting challenge.

\section{ACKNOWLEDGMENT}

The authors thank D. Neuhoff for recommending [50].

\section{REFERENCES}

[1] E. M. Haacke, R. W. Brown, M. R. Thompson, and R. Venkatesan, Magnetic Resonance Imaging: Physical Principles and Sequence Design. New York: Wiley, 1999.

[2] S. Ogawa, R. S. Menon, D. W. Tank, S. G. Kim, H. Merkle, J. M. Ellermann, and K. Ugurbil, "Functional brain mapping by blood oxygenation level-dependent contrast magnetic resonance imaging. A comparison of signal characteristics with a biophysical model," Biophys. J., vol. 64, no. 3, pp. 803-812, Mar. 1993.

[3] K. Sekihara, S. Matsui, and H. Kohno, "NMR imaging for magnets with large nonuniformities," IEEE Trans. Med. Imag., vol. MI-4, no. 4, pp. 193-199, Dec. 1985.

[4] E. Yudilevich and H. Stark, "Spiral sampling in magnetic resonance imaging - The effect of inhomogeneities," IEEE Trans. Med. Imag., vol. MI-6, no. 4, Dec. 1987.

[5] D. C. Noll, C. H. Meyer, J. M. Pauly, D. G. Nishimura, and A. Macovski, "A homogeneity correction method for magnetic resonance imaging with time-varying gradients," IEEE Trans. Med. Imag., vol. 10, no. 4, pp. 629-637, Dec. 1991.

[6] D. C. Noll, J. M. Pauly, C. H. Meyer, D. G. Nishimura, and A. Macovski, "Deblurring for non-2D fourier transform magnetic resonance imaging," Mag. Res. Med., vol. 25, pp. 319-333, 1992.

[7] H. Chang and J. M. Fitzpatrick, "A technique for accurate magnetic resonance imaging in the presence of field inhomogeneities," IEEE Trans. Med. Imag., vol. 11, no. 3, pp. 319-329, Sep. 1992.

[8] T. S. Sumanaweera, G. H. Glover, T. O. Binford, and J. R. Adler, "MR susceptibility misregistration correction," IEEE Trans. Med. Imag., vol. 12, no. 2, pp. 251-259, Jun. 1993.

[9] P. Jezzard and R. S. Balaban, "Correction for geometric distortion in echo planar images from $B_{0}$ field variations," Mag. Res. Med., vol. 34, no. 1, pp. 65-73, Jul. 1995.

[10] Y. M. Kadah and X. Hu, "Simulated phase evolution rewinding (SPHERE): A technique for reducing $B_{0}$ inhomogeneity effects in MR images," Mag. Res. Med., vol. 38, pp. 615-627, 1997.

[11] _ , "Algebraic reconstruction for magnetic resonance imaging under $B_{0}$ inhomogeneity," IEEE Trans. Med. Imag., vol. 17, no. 3, pp. 362-370, Jun. 1998.
[12] T. B. Harshbarger and D. B. Twieg, "Iterative reconstruction of singleshot spiral MRI with off-resonance," IEEE Trans. Med. Imag., vol. 18, no. 3, pp. 196-205, Mar. 1999.

[13] H. Schomberg, "Off-resonance correction of MR images," IEEE Trans. Med. Imag., vol. 18, no. 6, pp. 481-495, Jun. 1999.

[14] J. Kybic, P. Thevenaz, A. Nirkko, and M. Unser, "Unwarping of unidirectionally distorted EPI images," IEEE Trans. Med. Imag., vol. 19, no. 2, pp. $80-93$, Feb. 2000

[15] P. Munger, G. R. Crelier, T. M. Peters, and G. B. Pike, "An inverse problem approach to the correction of distortion in EPI images," IEEE Trans. Med. Imag., vol. 19, no. 7, pp. 681-689, Jul. 2000.

[16] K. S. Nayak and D. G. Nishimura, "Automatic field map generation and off-resonance correction for projection reconstruction imaging," Mag. Res. Med., vol. 43, no. 1, pp. 151-154, Jan. 2000.

[17] K. S. Nayak, C.-M. Tsai, C. H. Meyer, and D. G. Nishimura, "Efficient off-resonance correction for spiral imaging," Mag. Res. Med., vol. 45, no. 3, pp. 521-524, Mar. 2001.

[18] J. A. Akel, M. Rosenblitt, and P. Irarrazaval, "Off-resonance correction using an estimated linear time map," Mag. Res. Imag., vol. 20, no. 2, pp. 189-198, Feb. 2002.

[19] R. Deichmann, O. Josephs, C. Hutton, D. R. Corfield, and R. Turner, "Compensation of susceptibility-induced BOLD sensitivity losses in echo-planar fMRI imaging," NeuroImag., vol. 15, no. 1, pp. 120-135, Jan. 2002.

[20] H. Moriguchi, B. M. Dale, J. S. Lewin, and J. L. Duerk, "Block regional off-resonance correction (BRORC): A fast and effective deblurring method for spiral imaging," Mag. Res. Med., vol. 50, no. 3, pp. 643-648, Sep. 2003.

[21] B. P. Sutton, D. C. Noll, and J. A. Fessler, "Fast, iterative image reconstruction for MRI in the presence of field inhomogeneities," IEEE Trans. Med. Imag., vol. 22, no. 2, pp. 178-188, Feb. 2003.

[22] D. C. Noll, J. A. Fessler, and B. P. Sutton, "Conjugate phase MRI reconstruction with spatially variant sample density correction," IEEE Trans. Med. Imag., vol. 24, no. 3, pp. 325-336, Mar. 2005.

[23] B. P. Sutton, D. Noll, and J. A. Fessler, "Simultaneous estimation of image and inhomogeneity field map," in Proc. ISMRM Minimum Data Acquisition Workshop, 2001, pp. 15-18.

[24] B. P. Sutton, J. A. Fessler, and D. C. Noll, "Field-corrected imaging using joint estimation of image and field map," Proc. Int. Soc. Mag. Res. Med., p. 737, 2002.

[25] B. P. Sutton, D. C. Noll, and J. A. Fessler, "Dynamic field map estimation using a spiral-in/spiral-out acquisition," Mag. Res. Med., vol. 51, no. 6, pp. 1194-1204, Jun. 2004.

[26] S. Lee, S. J. Peltier, J. A. Fessler, and D. Noll, "Estimation of $R_{2}^{*}$ using extended rosette acquisition," in Hum. Brain Map, 2002, pp. 151-152.

[27] V. T. Olafsson, D. C. Noll, and J. A. Fessler, "New approach for estimating $\Delta R_{2}^{*}$ in fMRI," in Proc. Int. Soc. Mag. Res. Med., 2003, p. 132.

[28] O. Speck and J. Hennig, "Functional imaging by $I_{0}$ and $T_{2}^{*}$-parameter mapping using multi-image EPI," Mag. Res. Med., vol. 40, pp. 243-248, 1998.

[29] S. Lee, J. A. Fessler, and D. Noll, "A simultaneous estimation of field inhomogeneity and R2* maps using extended rosette trajectory," in Proc. Int. Soc. Mag. Res. Med., 2002, p. 2327.

[30] B. P. Sutton, S. J. Peltier, J. A. Fessler, and D. C. Noll, "Simultaneous estimation of $I_{0}, R_{2}^{*}$, and field map using a multi-echo spiral acquisition," in Proc. Int. Soc. Mag. Res. Med., 2002, p. 1323.

[31] S. J. Peltier, B. P. Sutton, J. A. Fessler, and D. C. Noll, "Simultaneous estimation of $I_{0}, R_{2}^{*}$, and field map using a multi-echo spiral acquisition," in Hum. Brain Map, 2002, pp. 95-96.

[32] H. Eggers, T. Schaeffter, B. Aldefeld, and P. Boesiger, "Combined $\Delta \mathrm{B} 0$ and $\mathrm{T} 2 *$ correction for radial multi-gradient-echo imaging," in Proc. Int. Soc. Mag. Res. Med., 2003, p. 478.

[33] D. B. Twieg, "Parsing local signal evolution directly from a single-shot MRI signal: A new approach for fMRI," Mag. Res. Med., vol. 50, no. 5, pp. 1043-1052, Nov. 2003.

[34] S. Lee, D. Noll, and J. A. Fessler, "EXTended Rosette ACquisition technique (EXTRACT): A dynamic R2* mapping method using extended rosette trajectory," in Proc. Int. Soc. Mag. Res. Med., 2004, p. 2128.

[35] V. Olafsson, J. A. Fessler, and D. C. Noll, "Dynamic update of R2* and field map in fMRI," in Proc. Int. Soc. Mag. Res. Med., 2004, p. 45.

[36] B. P. Sutton, J. A. Fessler, and D. Noll, "Iterative MR image reconstruction using sensitivity and inhomogeneity field maps," in Proc. Int. Soc. Mag. Res. Med., 2001, p. 771. 
[37] D. C. Noll, "Reconstruction Techniques for Magnetic Resonance Imaging," Ph.D. dissertation, Stanford Univ., Stanford, CA, 1991.

[38] K. P. Pruessmann, M. Weiger, P. Börnert, and P. Boesiger, "Advances in sensitivity encoding with arbitrary k-space trajectories," Mag. Res. Med., vol. 46, no. 4, pp. 638-651, Oct. 2001.

[39] K. Gröchenig and T. Strohmer, "Numerical and theoretical aspects of nonuniform sampling of band-limited images," in Nonuniform Sampling: Theory and Practice, F. Marvasti, Ed. New York: Kluwer, 2001, pp. 283-324.

[40] F. Wajer and K. P. Pruessmann, "Major speedup of reconstruction for sensitivity encoding with arbitrary trajectories," in Proc. Int. Soc. Mag. Res. Med., 2001, p. 767.

[41] H. Eggers and P. B., "Comparison of gridding- and convolution-based iterative reconstruction algorithms for sensitivity-encoded noncartesian acquisitions," in Proc. Int. Soc. Mag. Res. Med., 2002, p. 743.

[42] R. M. Lewitt and S. Matej, "Overview of methods for image reconstruction from projections in emission computed tomography," Proc. IEEE, vol. 91, no. 10, pp. 1588-1611, Oct. 2003.

[43] R. Van de Walle, H. H. Barrett, K. J. Myers, M. I. Altbach, B. Desplanques, A. F. Gmitro, J. Cornelis, and I. Lemahieu, "Reconstruction of MR images from data acquired on a general nonregular grid by pseudoinverse calculation," IEEE Trans. Med. Imag., vol. 19, no. 12, pp. 1160-1167, Dec. 2000.

[44] B. P. Sutton, D. C. Noll, and J. A. Fessler, "Compensating for withinvoxel susceptibility gradients in BOLD fMRI," in Proc. Int. Soc. Mag. Res. Med., 2004, p. 349.

[45] B. P. Sutton, "Physics-Based Reconstruction of Magnetic Resonance Images," Ph.D. dissertation, Univ. Michigan, Ann Arbor, MI, 2003.

[46] J. A. Fessler and B. P. Sutton, "Nonuniform fast Fourier transforms using min-max interpolation," IEEE Trans. Signal Process., vol. 51, no. 2, pp. 560-574, Feb. 2003.

[47] R. H. Chan and M. K. Ng, "Conjugate gradient methods for Toeplitz systems," SIAM Rev., vol. 38, no. 3, pp. 427-482, Sep. 1996.

[48] P. Irarrazabal, C. H. Meyer, D. G. Nishimura, and A. Macovski, "Inhomogeneity correction using an estimated linear field map," Mag. Res. Med., vol. 35, pp. 278-282, 1996.

[49] L.-C. Man, J. M. Pauly, and A. Macovski, "Multifrequency interpolation for fast off-resonance correction," Mag. Res. Med., vol. 37, pp. 785-792, 1997.

[50] A. Gersho, "Asymptotically optimal block quantization," IEEE Trans. Inf. Theory, vol. IT-25, no. 4, pp. 373-380, Jul. 1979.

[51] M. R. Willcott, G. L. Mee, and J. P. Chesick, "Magnetic field mapping in NMR imaging," Mag. Res. Med., vol. 5, no. 4, pp. 301-306, 1987.

[52] H. Schomberg and J. Timmer, "The gridding method for image reconstruction by Fourier transformation," IEEE Trans. Med. Imag., vol. 14, no. 3, pp. 596-607, Sep. 1995.

[53] C. Barnet, J. Tsao, and K. P. Pruessmann, "Efficient iterative reconstruction for MRI in strongly inhomogeneous $B_{0}$," in Proc. Int. Soc. Mag. Res. Med., 2004, p. 347.

[54] J. A. Fessler and D. C. Noll, "Iterative image reconstruction in MRI with separate magnitude and phase regularization," in Proc. IEEE Int. Symp. Biomed. Imag., 2004, pp. 209-212.

[55] J. A. Fessler and S. D. Booth, "Conjugate-gradient preconditioning methods for shift-variant PET image reconstruction," IEEE Trans. Imag. Process., vol. 8, no. 5, pp. 688-699, May 1999.

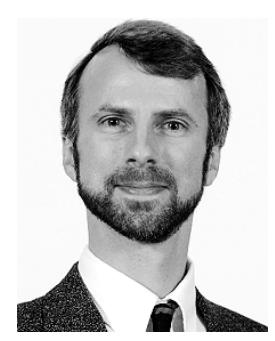

Jeffrey A. Fessler (M'90-SM'00) received the Ph.D. degree in electrical engineering in 1990 from Stanford University, Stanford, CA.

He has since been with the University of Michigan, Ann Arbor, first as a DoE Alexander Hollaender post-doctoral fellow and then as an Assistant Professor in the Division of Nuclear Medicine. Since 1995, he has been with the Electrical Engineering and Computer Science, Biomedical Engineering, and Radiology Departments. His research interests are in statistical aspects of imaging problems, and he has supervised doctoral research in PET, SPECT, X-ray CT, MRI, and optical imaging.

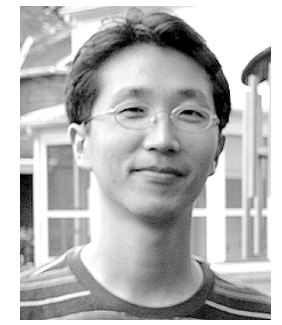

Sangwoo Lee (S'00) was born in Seoul, Korea, in 1973. He received the B.S.E. degree in electrical engineering from Seoul National University in 1996 and the M.S.E. degree from the Department of Electrical Engineering and Computer Science, University of Michigan, Ann Arbor, in 2001, where he is currently pursuing the Ph.D. degree in signal processing.

His research interests include statistical iterative magnetic resonance image reconstruction and MR pulse sequence design.

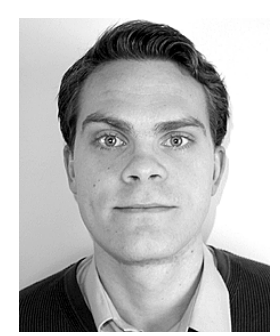

Valur T. Olafsson (S'05) received the B.S. degree in electrical engineering from the University of Iceland, Reykjavik, Iceland, in 2000 and the M.S. degree in electrical engineering from the University of Michigan, Ann Arbor, in 2003, where he is currently pursuing the $\mathrm{Ph} . \mathrm{D}$. degree.

His current research interests are in iterative MR image reconstruction methods.

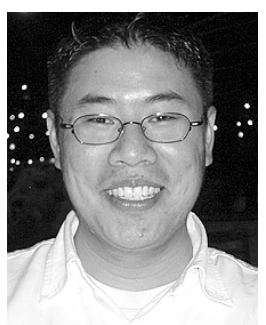

Hugo R. Shi (S'05) received the B.S. degree in electrical engineering and computer science from the University of California, Berkeley, in 2003.

$\mathrm{He}$ is currently a second-year graduate student at the University of Michigan, Ann Arbor, studying electrical engineering systems, with an emphasis in medical image processing. His current research interests include regularization design for statistical image reconstruction from three-dimensional PET scans.

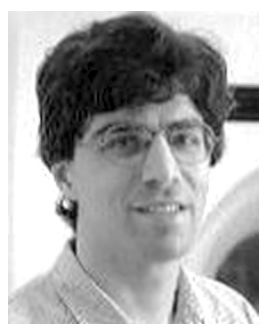

Douglas C. Noll (M'92) received the B.S. degree in electrical engineering from Bucknell University, Lewisburg, PA, in 1985 and the M.S. and Ph.D. degrees in electrical engineering from Stanford University, Stanford, CA, in 1986 and 1991, respectively.

Since 1998, he has been Associate Professor of biomedical engineering and radiology and Co-Director of the Functional MRI Laboratory at the University of Michigan, Ann Arbor. From 1991 to 1998, He was with the Department of Radiology at the University of Pittsburgh, Pittsburgh, PA. His expertise is in magnetic resonance image acquisition, reconstruction, and processing with a focus on functional brain imaging.

Dr. Noll is a member of the International Society for Magnetic Resonance in Medicine and is a Fellow of the American Institute for Medical and Biological Engineering. 